\title{
A Comment on Rotons in Two-Dimensional Liquid Helium II*
}

\author{
W. Götze \\ Physik-Department der Technischen Universität, and Max-Planck-Institut für Physik, \\ München, Germany \\ and M. Lücke
}

Department of Physics, Harvard University, Cambridge, Massachusetts

(Received May 10, 1976)

The spectrum of rotons in two-dimensional liquid helium II is estimated within the Bijl-Feynman theory. Due to stronger short-range order and more effective back flow, the roton gap $\Delta$ is found to be about half as big as the bulk roton energy.

The decrease of the superfluid density of liquid helium II near boundaries is experimentally well established. ${ }^{1,2}$ The momentum quantization in restricted geometry may account for this effect in some cases. ${ }^{3}$ It has been argued also that near a surface one should expect a change of the bulk elementary excitation spectrum ${ }^{4}$ leading to a change of the normal fluid density. There also could exist special elementary excitations traveling along the helium surface ${ }^{5}$ increasing the normal density.

This view was adopted recently by Chester and Eytel ${ }^{6}$ to interpret their liquid helium data. They first calculated the thickness of the solidified layer due to van der Waals attraction. There remained a surface contribution to the normal density of the liquid. The previously suggested mechanisms ${ }^{3-5}$ could not explain the data. Chester and Eytel demonstrated a good fit to the experiments on the basis of postulated surface rotons having a gap energy $\Delta \sim 4.5 \mathrm{~K}$.

The determination of the solid helium layer on the basis of bulk thermodynamics and not quite certain van der Waals forces is of course not compelling, nor is it quite satisfactory to base the hypothesis of new excitations on one type of experiment. On the other hand, the Monte Carlo

* Supported by grants of the Deutsche Forschungsgemeinschaft and, in part, by the U.S. National Science Foundation through Grants DMR73-02363 and DMR72-02977 A03.

\section{1}

(ङ) 1976 Plenum Publishing Corporation, 227 West 17th Street, New York, N.Y. 10011. No part of this publication may be reproduced, stored in a retrieval system, or transmitted, in any form or by any means, electronic, mechanical, photocopying, microfilming, recording, or otherwise, without written permission of the publisher. 
calculation of the excitation spectrum of two-dimensional helium systems carried out by Padmore ${ }^{7}$ shows, indeed, that the roton spectrum in films might be of a form similar to the one postulated by Chester and Eytel. Two-dimensional behavior of helium has also been found in experiments on specific heat, ${ }^{8}$ third sound, ${ }^{2,9}$ and isotherms ${ }^{10}$ of films adsorbed on exfoliated graphite.

In this note we want to present two simple arguments which lead to a rough estimation of the roton gap $\Delta$ in two dimensions. Feynman ${ }^{11}$ explained why the wave function ${ }^{12} \psi_{\mathbf{k}}=\rho_{\mathbf{k}} \psi$ describes fairly well zerotemperature excitations in Bose systems of high density and strong hardcore interaction. His reasoning is valid also for two-dimensional layers where $\psi$ is the correlated ground state and $\rho_{\mathbf{k}}$ is the density fluctuation operator for momenta parallel to the surface. This test function yields ${ }^{11,12}$ a variational estimate for the excitation energy

$$
\varepsilon(k)=k^{2} /[2 m s(k)]
$$

in terms of the liquid structure factor $s(k)=\left\langle\rho_{\mathbf{k}}^{*} \rho_{\mathbf{k}}\right\rangle$ and the particle mass $m$. Unfortunately, we are not aware of experimental or theoretical results concerning $s(k)$ for layers. We will find an estimate for the pair correlation function $g(r)$, which is the Fourier transform of $s(k)$, by treating the liquid as a randomized Einstein solid. ${ }^{13}$

Averaging the correlation function, e.g., of an fcc solid over the solid angle $4 \pi$ smears out the structure more efficiently than averaging a closepacked surface of the same solid over the angle $2 \pi$. Consequently, the correlation function $g(r)$ in two dimensions should exhibit more structure due to ordering than the structure factor of a three-dimensional liquid. As a result, $s_{3}(k)$ of the bulk is not as strongly peaked as $s_{2}(k)$ for a layer. Hence the roton minimum of Eq. (1) should be lower in two-dimensional helium than in bulk helium.

To get a quantitative formulation of the preceding reasoning, we start with the $n$ th-atom contribution to the pair correlation function of a threedimensional Einstein solid

$$
g_{\mathbf{n}}(\mathbf{r})=\left\{\exp \left[\left(\mathbf{r}-\mathbf{r}_{\mathbf{n}}\right)^{2} / 2 \xi_{n}^{2}\right]\right\}\left[(2 \pi)^{1 / 2} \xi_{n}\right]^{-3}
$$

Here $r_{n}$ is the nth-atom position and $\xi_{n}$ is the characteristic width. Averaging $g_{\mathbf{m}}(\mathbf{r})$ over the solid angle $4 \pi$, one gets the contribution of the $N$ th-neighbor shell

$$
\begin{aligned}
g_{N}^{(3)}(r)= & Z_{N}^{3}\left\{\exp \left[-\left(r-r_{N}\right)^{2} / 2 \xi_{N}^{2}\right]-\exp \left[-\left(r+r_{N}\right)^{2} / 2 \xi_{N}^{2}\right]\right\} \\
& {\left[4 \pi(2 \pi)^{1 / 2} \xi_{N} r_{N} r\right]^{-1} }
\end{aligned}
$$

Here $Z_{N}^{3}$ is the number of atoms in the $N$ th shell and $r_{N}$ and $\xi_{N}$ are the 
corresponding radius and width. The total structure factor is obtained as

$$
s_{3}(k)-1=4 \pi \int_{0}^{\infty} d r{ }^{2} \frac{\sin k r}{k r}\left[\sum_{N} g_{N}^{(3)}(r)-n^{(3)}\right]
$$

The $r_{N}$ are given for primitive lattices by the lattice constant. We choose it such that the density of the solid is the same as the experimental density $n^{(3)}$ of liquid helium.

In solids $\xi_{N}$ would be independent of $N$. In liquids the absence of long-range correlations can be approximated by the diffusion ansatz ${ }^{13}$ $\xi_{N}=A\left(r_{N} / r_{1}\right)^{1 / 2}$. The quantity $A$, which we use as a fit parameter, is fixed by requiring $s_{3}(k)$ in (4) to have the same peak value as the experimental structure factor. ${ }^{14}$ The resulting $s_{3}(k)$ for an underlying fcc crystal $\left(r_{1}=\right.$ $4.0 \AA, A=1.1 \AA)$ is shown in Fig. 1 . We have not evaluated $s_{3}(k)$ for the nonprimitive hcp structure. Since $Z_{1}, Z_{2}, r_{1}$, and $r_{2}$ are the same in both close-packed structures, one cannot expect remarkable differences. The result for a bcc lattice differs from the fcc approximation only by a few percent. Figure 1 shows that our approximate structure factor $s_{3}(k)$ [Eq. (4)] is quite similar to the experimental one,${ }^{14}$ but the position of the maximum is too low by about $10 \%$.

Now let us consider a close-packed surface of the above-described fcc lattice. Averaging $g_{\mathbf{n}}(\mathbf{r})$ over the angle $2 \pi$, one finds as contribution of the $N$ th-neighbor shell in two dimensions

$$
g_{N}^{(2)}(r)=Z_{N}^{2}\left\{\exp \left[-\left(r^{2}+r_{N}^{2}\right) / 2 \xi_{N}^{2}\right]\right\} I_{0}\left(r_{N} / \xi_{N}^{2}\right) / 2 \pi \xi_{N}^{2}
$$

The number of atoms in the $n$ th-neighbor ring is $Z_{N}^{2} ; r_{N}$ and $\xi_{N}$ have the same meaning as before and $I_{0}$ denotes a Bessel function of imaginary argument. ${ }^{15}$ The two-dimensional structure factor then reads

$$
s_{2}(k)-1=2 \pi \int_{0}^{\infty} r d r J_{0}(k r)\left[\sum_{N} g_{N}^{(2)}(r)-n^{(2)}\right]
$$

Here $n^{(2)}$ is the surface density and $J_{0}$ is an ordinary Bessel function. ${ }^{15}$ The result $s_{2}(k)$ is shown in Fig. 1. The oscillations in $s_{2}(k)$ are more pronounced than those in $s_{3}(k)$. In particular the maximum of the structure factor in two dimensions is higher by about $30 \%$ than the maximum in $s_{3}(k)$. So the roton minimum according to the Bijl-Feynman formula (1) is deeper on the surface than in bulk, as shown in Fig. 2.

Compared to experiment, the roton gap $\Delta$ according to Eq. (1) is much too large. This is caused by neglecting back flow effects, ${ }^{16}$ which are not incorporated into the Bijl wave function. The moving roton should be surrounded by a velocity field of the liquid, so that there are no large density fluctuations. Effectively this leads to an increase of the effective mass of the 


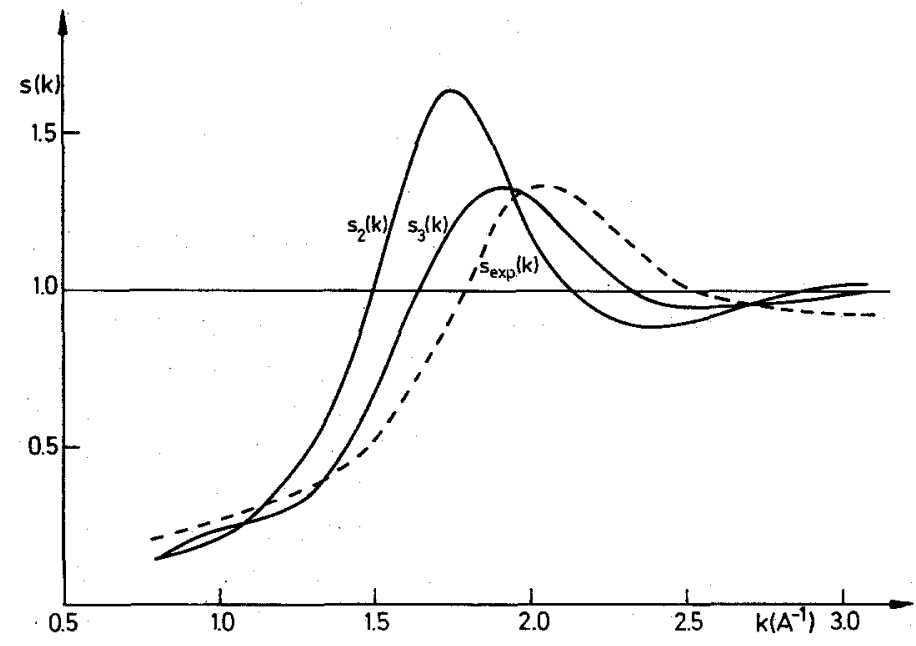

Fig. 1. Structure factors as function of momentum. $s_{\text {exp }}(k)$ is the experimental result ${ }^{14}$ for bulk helium, $s_{3}(k)$ is the estimate for three dimensions, and $s_{2}(k)$ is the estimate for two dimensions.

particles, which for short wavelengths can be estimated on the basis of classical hydrodynamics. ${ }^{17}$

In three dimensions a moving sphere is surrounded by a velocity field derived from the dipole potential $\phi \propto \nabla(1 / r)$. For large distances the velocity of the back flow decreases proportional to $1 / r^{3}$. This yields ${ }^{18}$ an effective mass $m_{3}^{*}=(3 / 2) m$. Substitution of this value into Eq. (1) yields the roton curve shown in Fig. 2 (lower, dotted curve). The roton minimum is $10.2 \mathrm{~K}$, an estimate not too far from the experimental result of $9 \mathrm{~K}$.

In two dimensions, on the other hand; the velocity field is derived from a logarithmic potential $\phi \propto(\log r) .^{18}$ The velocity decreases proportional to $1 / r^{2}$. There is relatively more mass in motion in two than in three dimensions, leading to an effective mass $m_{2}^{*}=2 m \cdot{ }^{17,18}$ Substitution of this result into Eq. (1) yields the lowest curve in Fig. 2.

In conclusion, one arrives at the statement that the combined effect of stronger short-range order and more effective back flow yields surface rotons whose minimum compared to the bulk roton minimum has about a $10 \%$ lower momentum and about half the energy. This conclusion, incidentally, is in quantitative agreement with the statements of Chester and Eytel. 


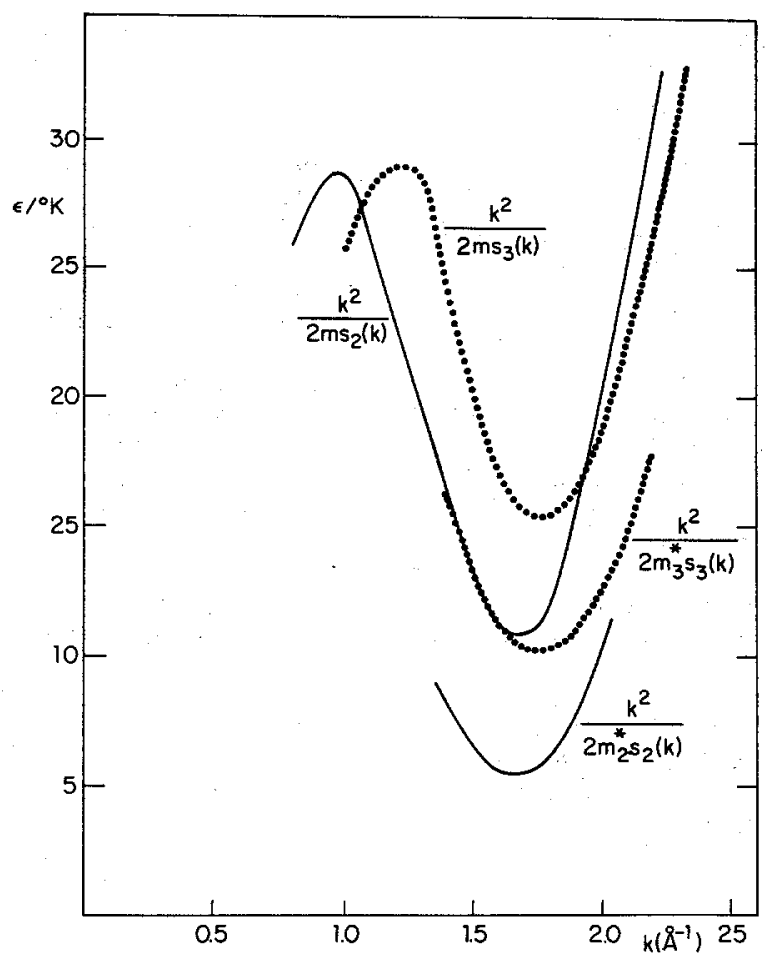

Fig. 2. Excitation spectra according to the Bijl-Feynman formula for two and three dimensions (dotted). The effective masses are $m_{3}^{*}=\frac{3}{2} m$ and $m_{2}^{*}=2 m$.

\section{ACKNOWLEDGMENTS}

We thank Profs. Marvin Chester, P. C. Martin, and R. Orbach for discussions as well as for the kind hospitality offered to us at the University of California, Los Angeles, and at Harvard University, respectively, during the time the preceding note was written.

\section{REFERENCES}

1. F. D. M. Pobell, H. W. Chan, L. R. Corruccini, R. P. Henkel, S. W. Schwenterly, and J. D. Reppy, Phys. Rev. Lett. 28, 542 (1972); M. Chester and L. C. Yang, Phys. Rev. Lett. 31, 1377 (1973).

2. J. H. Scholtz, E. O. McLean, and I. Rudnick, Phys. Rev. Lett. 32, 147 (1974); T. E. Washburn, J. E. Rutledge, and J. M. Mochel, Phys. Rev. Lett. 34, 183 (1975). 
3. T. C. Padmore, Phys. Rev, Lett. 28, 1512 (1972); G. Haug, J. Low Temp. Phys. 12, 479 (1973); T. C. Padmore and J. D. Reppy, Phys. Rev. Lett. 33, 1410 (1974).

4. C. G. Kuper, Physica 24, 1009 (1958).

5. L. S. Rent and I. Z. Fisher, Zh. Eksp. Teor. Fiz. 60, 1814 (1971); A. F. Andreev and D. A. Kompaneets, Zh. Eksp. Teor. Fiz. 61, 2459 (1971); D. O. Edwards, J. R. Eckhardt, and F. M. Gasparini, Phys. Rev. A 9, 2070 (1964).

6. Marvin Chester and L. Eytel, Phys. Rev. B 13, 1069 (1976).

7. T. C. Padmore, Phys. Rev. Lett. 32, 826 (1974).

8. D. C. Hickernell, E. O. McLean, and O. E. Vilches, Phys. Rev. Lett. 28, 789 (1972); M. Bretz, J. G. Dash, D. C. Hickernell, E. O. McLean, and O. E. Vilches, Phys. Rev. A 8, 1589 (1973).

9. D. J. Bishop, J. M. Parpia, and J. D. Reppy, in Proc. 14th Int. Conf. Low Temp. Phys., M. Krusius and M. Vuorio, eds. (North-Holland, Amsterdam, 1975), Vol. 1, p. 380.

10. G. J. Coellner, J. G. Daunt, and E. Lerner, J. Low Temp. Phys. 21, 347 (1975); A. D. Navaco, J. Low Temp. Phys. 21, 359 (1975).

11. R. P. Feynman, Phys. Rev. 91, 1291, 1301 (1953); 94, 262 (1954).

12. A. Bijl, Physica 7, 869 (1940).

13. J. Frenkel, Kinetic Theory of Liquids (Dover, New York, 1955), Chapter III, $\$ 4$.

14. E. K. Achter and L. Meyer, Phys. Rev. 188, 291 (1969).

15. M. Abramowitz and I. A. Stegun, Handbook of Mathematical Functions (Dover, New York).

16. R. P. Feynman, In Progress in Low Temp. Phys. I, C. J. Gorter, ed. (North-Holland Publishing, Amsterdam, 1955), p. 17.

17. W. Brenig, Z. Phys. 144, 488 (1956).

18. L. D. Landau and E. M. Lifshitz, Fluid Mechanics (Pergamon Press, Oxford, 1959), Chapter I, $\$ \$ 10,11$. 